Journal of Applied AnAlysis

Vol. 14, No. 1 (2008), pp. 53-61

\title{
UPPER AND LOWER SOLUTIONS METHOD FOR FOURTH-ORDER PERIODIC BOUNDARY VALUE PROBLEMS
}

\author{
S. WENG, H. GAO, D. JIANG and X. HOU \\ Received December 28, 2005 and, in revised form, October 19, 2007
}

\begin{abstract}
The purpose of this paper is to prove the existence of a solution of the following periodic boundary value problem

$$
\left\{\begin{array}{l}
u^{(4)}(t)=f\left(t, u(t), u^{\prime \prime}(t)\right), \quad t \in[0,2 \pi] \\
u(0)=u(2 \pi), u^{\prime}(0)=u^{\prime}(2 \pi), u^{\prime \prime}(0)=u^{\prime \prime}(2 \pi), u^{\prime \prime \prime}(0)=u^{\prime \prime \prime}(2 \pi)
\end{array}\right.
$$

in the presence of an upper solution $\beta$ and a lower solution $\alpha$ with $\beta \leq \alpha$, where $f(t, u, v)$ satisfies one side Lipschitz condition.
\end{abstract}

\section{INTRODUCTION AND MAIN RESULTS}

In this paper, we study a fourth-order periodic boundary value problem of the form

2000 Mathematics Subject Classification. Primary: 34B15, 34B16.

Key words and phrases. Periodic boundary value problem, existence, upper and lower solutions method.

Project Supported by the National Natural Science Foundation of P. R. China (No. 10171010).

ISSN 1425-6908（C) Heldermann Verlag. 


$$
\left\{\begin{array}{l}
u^{(4)}(t)=f\left(t, u(t), u^{\prime \prime}(t)\right), \quad t \in[0,2 \pi] \\
u(0)=u(2 \pi), u^{\prime}(0)=u^{\prime}(2 \pi), u^{\prime \prime}(0)=u^{\prime \prime}(2 \pi), u^{\prime \prime \prime}(0)=u^{\prime \prime \prime}(2 \pi),
\end{array}\right.
$$

where $f(t, u, v)$ is a Carathéodory function.

A function $f:[0,2 \pi] \times \mathbb{R}^{2} \rightarrow \mathbb{R}$ is said to be a Carathéodory function if it possesses the following three properties:

(i) For all $(u, v) \in \mathbb{R}^{2}$, the function $t \rightarrow f(t, u, v)$ is measurable on $[0,2 \pi]$.

(ii) For almost all $t \in[0,2 \pi]$, the function $(u, v) \rightarrow f(t, u, v)$ is continuous on $\mathbb{R}^{2}$.

(iii) For any given $N>0$, there exists $g_{N}(t)$, a Lebesgue integrable function defined on $[0,2 \pi]$ such that

$$
|f(t, u, v)| \leq g_{N}(t) \text { for a.e. } t \in[0,2 \pi],
$$

whenever $|u|,|v| \leq N$.

To develop a monotone method, we need the concepts of upper and lower solutions. We say that $\beta \in W^{4,1}[0,2 \pi]$ is an upper solution to the problem (1.1), if it satisfies

$$
\left\{\begin{array}{l}
\beta^{(4)}(t) \leq f\left(t, \beta(t), \beta^{\prime \prime}(t)\right), \quad t \in[0,2 \pi] \\
\beta(0)=\beta(2 \pi), \beta^{\prime}(0)=\beta^{\prime}(2 \pi), \beta^{\prime \prime}(0)=\beta^{\prime \prime}(2 \pi), \beta^{\prime \prime \prime}(0) \leq \beta^{\prime \prime \prime}(2 \pi) .
\end{array}\right.
$$

Similarly, a function $\alpha \in W^{4,1}[0,2 \pi]$ is said to be a lower solution to (1.1), if it satisfies

$$
\left\{\begin{array}{l}
\alpha^{(4)}(t) \geq f\left(t, \alpha(t), \alpha^{\prime \prime}(t)\right), \quad t \in[0,2 \pi] \\
\alpha(0)=\alpha(2 \pi), \alpha^{\prime}(0)=\alpha^{\prime}(2 \pi), \alpha^{\prime \prime}(0)=\alpha^{\prime \prime}(2 \pi), \alpha^{\prime \prime \prime}(0) \geq \alpha^{\prime \prime \prime}(2 \pi) .
\end{array}\right.
$$

We call a function $u \in W^{4,1}[0,2 \pi]$ a solution to the problem (1.1), if it is an upper and a lower solution to (1.1).

Recently, the equation of (1.1) with non-periodic boundary value problems has been studied by several authors, for examples, see [1], [3], [4], [6]-[8], [10], [12]-[14], [16], [18]. In [1], [6]-[8], [16], [18], all of the results are based upon the the Leray-Schauder continuation method and topological degree. In [3], [4], [10], [13], [14], the upper and lower solutions method has been studied when $f=f(t, u)$. In [12], the authors have studied the existence of the methods of lower and upper solutions and the monotone iterative technique.

Only a few have dealt with the periodic boundary value problem (1.1) (see [2], [9], [15], [17]). When $f=f(t, u)$, the authors of $[2,15]$, have studied the problem by the methods of lower and upper solutions and the monotone iterative technique. Wang [17] has investigated a special case of (1.1) (where $f(t, u, v)=k v+F(t, u)$ ) in the presence of a lower solution $\alpha(t)$ and an upper solution $\beta(t)$ with $\beta(t) \leq \alpha(t)$. Recently, Jiang, Gao and Wan [9] have dealt with (1.1) by means of a monotone iterative technique 
in the presence of a lower solution $\alpha(t)$ and an upper solution $\beta(t)$ with $\beta(t) \leq \alpha(t)$. To develop a monotone method, the following hypotheses are needed in [9]:

(A1) For any given $\beta, \alpha \in C[0,2 \pi]$ with $\beta(t) \leq \alpha(t)$ on $[0,2 \pi]$, there exist $0<A \leq B$ such that

$$
A\left(v_{2}-v_{1}\right) \leq f\left(t, u, v_{2}\right)-f\left(t, u, v_{1}\right) \leq B\left(v_{2}-v_{1}\right)
$$

for a.e. $t \in[0,2 \pi]$ whenever $\beta(t) \leq u \leq \alpha(t), v_{1}, v_{2} \in \mathbb{R}$, and $v_{1} \leq v_{2}$.

(A2) Inequality

$$
f\left(t, u_{2}, v\right)-f\left(t, u_{1}, v\right) \geq-\frac{A^{2}}{4}\left(u_{2}-u_{1}\right)
$$

holds for a.e. $t \in[0,2 \pi]$, whenever $\beta(t) \leq u_{1} \leq u_{2} \leq \alpha(t), v \in \mathbb{R}$.

The purpose of this paper is to give the existence result of solution of (1.1) under the assumption that there exist a lower solution $\alpha(t)$ and an upper solution $\beta(t)$ of (1.1) with $\beta(t) \leq \alpha(t)$ and $f(t, u, v)$ only satisfies one side Lipschitz condition. We develop the upper and lower solutions method and prove that the solution $u(t)$ of $(1.1)$ satisfies $\beta(t) \leq u(t) \leq \alpha(t)$. Our result extends and complements those in [2], [5], [9], [15], [17].

To develop upper and lower solutions method, we need the following hypotheses:

(H1) For any given $\beta, \alpha \in C[0,2 \pi]$ with $\beta(t) \leq \alpha(t)$ on $[0,2 \pi]$, there exist $A>0$ and $B>0$ such that $B^{2} \geq 4 A$ and

$$
f\left(t, u_{2}, v_{2}\right)-f\left(t, u_{1}, v_{1}\right) \geq-A\left(u_{2}-u_{1}\right)+B\left(v_{2}-v_{1}\right)
$$

for a.e. $t \in[0,2 \pi]$ whenever $\beta(t) \leq u_{1} \leq u_{2} \leq \alpha(t), v_{1}, v_{2} \in \mathbb{R}$, and $v_{1} \leq v_{2}$.

Let $m<0$ and $M<0$ are two roots to the equation $x^{2}+B x+A=0$, then

$$
m+M=-B, \quad m M=A .
$$

Let

$$
A(t):=\alpha^{\prime \prime}(t)+m \alpha(t), \quad B(t):=\beta^{\prime \prime}(t)+m \beta(t) .
$$

The main result of this paper is stated as follows.

Theorem 1. Suppose that there exists a lower solution $\alpha(t)$ and an upper solution $\beta(t)$ of (1.1) such that $\beta(t) \leq \alpha(t)$ on $[0,2 \pi]$, and $f(t, u, v)$ is a Carathéodory function satisfying the hypotheses $(H 1)$ : there exist $a>0$ and $b>0$ such that $b^{2} \geq 4 a$ and

$$
f\left(t, u_{2}, v_{2}\right)-f\left(t, u_{1}, v_{1}\right) \geq-a\left(u_{2}-u_{1}\right)+b\left(v_{2}-v_{1}\right) .
$$


Then $A(t) \leq B(t)$ on $[0,2 \pi]$ and (1.1) has one solution $u \in W^{4,1}[0,2 \pi]$ such that

$$
\beta(t) \leq u(t) \leq \alpha(t), \quad A(t) \leq u^{\prime \prime}(t)+m u(t) \leq B(t) .
$$

\section{Proof of Theorem 1}

Lemma 1 (Maximum principle). Let $y \in W^{2,1}[0,2 \pi]$, and satisfies

$$
\left\{\begin{array}{l}
y^{\prime \prime}(t)-k y(t) \geq 0 \quad \text { for a.e. } t \in[0,2 \pi], \\
y(0)=y(2 \pi), \quad y^{\prime}(0) \geq y^{\prime}(2 \pi)
\end{array}\right.
$$

where $k>0$. Then $y(t) \leq 0$ on $[0,2 \pi]$.

Lemma 2. Suppose that there exists a lower solution $\alpha(t)$ and an upper solution $\beta(t)$ of $(1.1)$ such that $\beta(t) \leq \alpha(t)$ on $[0,2 \pi]$, and $f(t, u, v)$ is a Carathéodory function satisfying the hypotheses $(H 1)$. Then $A(t) \leq B(t)$ on $[0,2 \pi]$.

Proof. It follows from (1.2) and (1.3) that

$$
\begin{aligned}
& \left\{\begin{array}{l}
A^{\prime \prime}(t)+M A(t) \geq f(t, \alpha(t), A(t)-m \alpha(t))+(m+M) A(t)-m^{2} \alpha(t), \\
t \in[0,2 \pi] \\
A(0)=A(2 \pi), A^{\prime}(0) \geq A^{\prime}(2 \pi),
\end{array}\right. \\
& \left\{\begin{array}{c}
B^{\prime \prime}(t)+M B(t) \leq f(t, \beta(t), B(t)-m \beta(t))+(m+M) B(t)-m^{2} \beta(t), \\
t \in[0,2 \pi] \\
B(0)=B(2 \pi), B^{\prime}(0) \leq B^{\prime}(2 \pi) .
\end{array}\right.
\end{aligned}
$$

Let $y(t)=A(t)-B(t)$, then $y(0)=y(2 \pi), y^{\prime}(0) \geq y^{\prime}(2 \pi)$.

Assume that $y(t)>0$ for some $t \in[0,2 \pi]$. Since $y(t)$ is a continuous function defined on a closes interval $[0,2 \pi]$, it can attain its maximum value on $[0,2 \pi]$.

If $\max y(t)=y\left(t_{0}\right)>0$ where $t_{0} \in(0,2 \pi)$, then there is an interval $[c, d] \subseteq(0,2 \pi)$ such that $y(c)=y(d)$ and $y(t)>0$ in $[c, d]$, it follows from $\left(H_{1}\right)$ that

$$
\begin{aligned}
y^{\prime \prime}+M y(t) \geq & f(t, \alpha(t), A(t)-m \alpha(t))-f(t, \beta(t), B(t)-m \beta(t)) \\
& +(m+M) y(t)-m^{2}(\alpha(t)-\beta(t)) \\
\geq & -\left(A+B m+m^{2}\right)(\alpha(t)-\beta(t))+(B+m+M) y(t) \\
= & 0, \quad t \in[c, d],
\end{aligned}
$$

then by Lemma 1 , we have $y(t) \leq 0$ on $[c, d]$, which is a contradiction. 
If $\max y(t)=y(0)=y(2 \pi)>0$, and hence there exists $s \in(0,2 \pi)$ with $y(s)>0$, then there would be $0 \leq a<s<b \leq 2 \pi$ such that $y(t)>0$ in $(a, b)$ with $y(a)=y(b)=0$. By (1.2) and (1.3), we have

$$
y^{\prime \prime}(t)+M y(t) \geq 0, \quad t \in[a, b], \quad y(a)=y(b)=0 .
$$

This leads to $y(t) \leq 0$ on $[a, b]$, which is again a contradiction.

If $y(0)>0$ (then $y(2 \pi)=y(0)>0$ ), then there would be $a<b$ in $(0,2 \pi)$ such that $y(t)>0$ on $[0, a) \cup(b, 2 \pi]$ with $y(a)=y(b)=0, y^{\prime}(a) \leq 0$, $y^{\prime}(b) \geq 0$. So we have $y^{\prime \prime}(t)+M y(t) \geq 0$ on $[0, a) \cup(b, 2 \pi]$, hence $y^{\prime}(t)$ is strictly increasing in $[0, a) \cup(b, 2 \pi]$, which implies that $y^{\prime}(0)<y^{\prime}(a) \leq 0 \leq$ $y^{\prime}(b)<y^{\prime}(2 \pi)$, this is a contradiction with the boundary conditions.

The proof of Lemma 2 is completed.

Lemma 3. If $m<0$, then for any $q(t) \in L^{1}[0,2 \pi]$, the problem

$$
\left\{\begin{array}{l}
u^{\prime \prime}+m u(t)=q(t), \quad \text { for a.e. } t \in[0,2 \pi] \\
u(0)=u(2 \pi), \quad u^{\prime}(0)=u^{\prime}(2 \pi),
\end{array}\right.
$$

has a unique solution $u \in W^{2,1}[0,2 \pi]$, and

$$
u(t)=L^{-1} q(t)=\int_{0}^{2 \pi} G_{m}(t, s) q(s) d s,
$$

where $\rho=\sqrt{-m}$ and

$$
G_{m}(t, s):= \begin{cases}-\frac{e^{\rho(t-s)}+e^{\rho(2 \pi-t+s)}}{2 \rho\left(e^{2 \rho \pi}-1\right)}, & 0 \leq s \leq t \leq 2 \pi, \\ -\frac{e^{\rho(s-t)}+e^{\rho(2 \pi-s+t)}}{2 \rho\left(e^{2 \rho \pi}-1\right)}, & 0 \leq t \leq s \leq 2 \pi .\end{cases}
$$

Proof. Let

$$
p(t, x)= \begin{cases}A(t), & x<A(t) \\ x, & A(t) \leq x \leq B(t) \\ B(t), & x>B(t)\end{cases}
$$

By Lemma 1, we have

$$
\alpha(t)=L^{-1} A(t), \quad \beta(t)=L^{-1} B(t), \quad \beta(t) \leq L^{-1} p(t, x) \leq \alpha(t) .
$$

Now we consider the following modified problem

$$
\left\{\begin{aligned}
x^{\prime \prime}+M x(t)= & f\left(t, L^{-1} p(t, x(t)),\left(I-m L^{-1}\right) p(t, x(t))\right) \\
& +(m+M) p(t, x(t))-m^{2} L^{-1} p(t, x(t)), \\
x(0)=x(2 \pi), \quad & x^{\prime}(0)=x^{\prime}(2 \pi) .
\end{aligned}\right.
$$


For each $x \in C[0,2 \pi]$,we define the mapping $\Phi: C[0,2 \pi] \rightarrow C[0,2 \pi]$

$$
(\Phi x)(t)=\int_{0}^{2 \pi} G_{M}(t, s)(F x)(s) d s
$$

where

$$
\begin{aligned}
(F x)(t):= & f\left(t, L^{-1} p(t, x(t)),\left(I-m L^{-1}\right) p(t, x(t))\right) \\
& +(m+M) p(t, x(t))-m^{2} L^{-1} p(t, x(t)) .
\end{aligned}
$$

Since $p(t, x(t))$ and $L^{-1} p(t, x(t))$ are bounded and $f(t, u, v)$ is a Carathéodory function, there exists $g(t)$, a Lebesgue integrable function defined on $[0,2 \pi]$ such that

$$
|(F x)(t)| \leq g(t) \text { for a. e. } t \in[0,2 \pi] .
$$

Thus $(\Phi x)(t)$ is also bounded.

We can easily prove that $\Phi: C[0,2 \pi] \rightarrow C[0,2 \pi]$ is completely continuous. Then Leray-Schauder fixed point Theorem assures that $\Phi$ has a fixed point $x \in C[0,2 \pi]$ and

$$
x(t)=\int_{0}^{2 \pi} G_{M}(t, s)(F x)(s) d s,
$$

thus the modified problem $(2.1)$ has one solution $x \in W^{2,1}[0,2 \pi]$. The proof of Lemma 3 is completed.

Lemma 4. Suppose that (H1) holds. Assume that $\alpha(t)$ and $\beta(t)$ are lower and upper solutions to (1.1) and $\beta(t) \leq \alpha(t)$ on $[0,2 \pi]$. Let $x \in W^{2,1}[0,2 \pi]$ be a solution to $(2.1)$, then $A(t) \leq x(t) \leq B(t)$ on $[0,2 \pi]$.

Remark 1. Lemma 4 implies $u(t)=L^{-1} x(t)=\int_{0}^{2 \pi} G_{m}(t, s) x(s) d s$ is a solution to $(1.1)$, since $u^{\prime \prime}+m u(t)=x(t), u(0)=u(2 \pi), u^{\prime}(0)=u^{\prime}(2 \pi)$ and $A(t) \leq x(t) \leq B(t)$.

Proof of Lemma 4. Since $\alpha(t)=L^{-1} A(t), \beta(t)=L^{-1} B(t)$,

$$
\left\{\begin{aligned}
B^{\prime \prime}(t)+M B(t) \leq & f\left(t, L^{-1} B(t),\left(I-m L^{-1}\right) B(t)\right)-m^{2} L^{-1} B(t) \\
& +(m+M) B(t), \\
B(0)=B(2 \pi), \quad & B^{\prime}(0) \leq B^{\prime}(2 \pi),
\end{aligned}\right.
$$

and

$$
\left\{\begin{aligned}
A^{\prime \prime}(t)+M A(t) \geq & f\left(t, L^{-1} A(t),\left(I-m L^{-1}\right) A(t)\right)-m^{2} L^{-1} A(t) \\
& +(m+M) A(t), \\
A(0)=A(2 \pi), \quad & A^{\prime}(0) \geq A^{\prime}(2 \pi) .
\end{aligned}\right.
$$


Let $y(t)=x(t)-B(t)$ and $z(t)=A(t)-x(t)$, then

$$
y(0)=y(2 \pi), \quad y^{\prime}(0) \geq y^{\prime}(2 \pi)
$$

and

$$
z(0)=z(2 \pi), \quad z^{\prime}(0) \geq z^{\prime}(2 \pi) .
$$

Applying an analogous approach used in the proof of Lemma 2, we can show that $y(t) \leq 0$ and $g(t) \leq 0$, and that is, $A(t) \leq x(t) \leq B(t)$.

The proof of Lemma 4 is completed.

By Remark 1, we have the results of Theorem 1.

\section{EXAMPLE}

In this section, we consider the periodic boundary value problem:

$$
\left\{\begin{array}{l}
u^{(4)}(t)-k u^{\prime \prime}(t)=F(t, u), \quad t \in[0,2 \pi] \\
u(0)=u(2 \pi), u^{\prime}(0)=u^{\prime}(2 \pi), u^{\prime \prime}(0)=u^{\prime \prime}(2 \pi), u^{\prime \prime \prime}(0)=u^{\prime \prime \prime}(2 \pi),
\end{array}\right.
$$

where $F(t, u)$ is a Carathéodory function, $k>0$.

To develop upper and lower solutions method, we also need the following hypothesis:

$(H)$ For any given $\beta, \alpha \in C[0,2 \pi]$ with $\beta(t) \leq \alpha(t)$ on $[0,2 \pi]$, inequality

$$
F\left(t, u_{2}\right)-F\left(t, u_{1}\right) \geq-\frac{k^{2}}{4}\left(u_{2}-u_{1}\right)
$$

holds for a.e. $t \in[0,2 \pi]$, whenever $\beta(t) \leq u_{1} \leq u_{2} \leq \alpha(t)$.

Let $A=k^{2} / 4, B=k$, then ( $\left.H 1\right)$ holds. Hence the conclusions of Theorem 1 hold. Then

$$
\alpha^{\prime \prime}(t)-\frac{k}{2} \alpha(t) \leq \beta^{\prime \prime}(t)-\frac{k}{2} \beta(t)
$$

and problem (3.1) has one solution $u \in W^{4,1}[0,2 \pi]$ such that

$$
\beta(t) \leq u(t) \leq \alpha(t), \quad \alpha^{\prime \prime}(t)-\frac{k}{2} \alpha(t) \leq u^{\prime \prime}(t)-\frac{k}{2} u(t) \leq \beta^{\prime \prime}(t)-\frac{k}{2} \beta(t) .
$$

In [17], Wang studied the problem (4.1) when $F$ is continuous on $[0,2 \pi] \times$ $\mathbb{R}$. In $[9,17]$, the authors obtained one solution $u \in W^{4,1}[0,2 \pi]$ of $(3.1)$ such that $\beta(t) \leq u(t) \leq \alpha(t)$.

We have improved the results of $[9,17]$. 


\section{References}

[1] Aftabizadeh, A. R., Existence and uniqueness theorems for fourth-order boundary value problems, J. Math. Anal. Appl. 116 (1986), 415-426.

[2] Cabada, A., The method of lower and upper solutions for second, third, fourth, and higher order boundary value problems, J. Math. Anal. Appl. 185 (1994), 302-320.

[3] De Coster, C., Sanchez, L., Upper and lower solutions, Ambrosetti-Prodi problems and positive solutions for fourth order O.D.E., Riv. Mat. Pura Appl. 14 (1994), 1129-1138.

[4] Dunninger, D., Existence of positive solutions for fourth-order nonlinear problems, Boll. Un. Mat. Ital. B (7) 1(4) (1987), 1129-1138.

[5] Gao, H., Weng, S., Jiang, D., Hou, X., On second order periodic boundary value problems with upper and lower solutions in the reversed order, Electron. J. Differential Equations 2006(25) (2006), 8 pp.

[6] Gupta, C. P., Existence and uniqueness results for a bending of an elastic beam equation at resonance, J. Math. Anal. Appl. 135 (1988), 208-225.

[7] Gupta, C. P., Existence and uniqueness theorem for a bending of an elastic beam equation, Appl. Anal. 26 (1988), 289-304.

[8] Gupta, C. P., Existence and uniqueness results for some fourth order fully quasilinear boundary value problems, Appl. Anal. 36 (1990), 169-175.

[9] Jiang, D. Q., Gao, W. J., Wan, A. Y., A monotone method for constructing extremal solutions to fourth-order periodic boundary value problems, Appl. Math. Comput. 132 (2002), 411-421.

[10] Korman, P., A maximum principle for fourth-order ordinary differential equations, Appl. Anal. 33 (1989), 267-273.

[11] Ladde, G. S., Lakshmikantham, V., Vatsala, A. S., Monotone Iterative Techniques for Nonlinear Differential Equations, Pitman (Advanced Publishing Program), Boston, MA, 1985.

[12] Ma, R. Y., Zhang, J. H., Fu, S. M., The method of lower and upper solutions for fourth-order two-point boundary value problems, J. Math. Anal. Appl. 215 (1997), 415-422.

[13] Sadyrabaev, F., A two-point boundary value problem for a fourth-order equation (Russian), Latv. Univ. Zināt Raksti 553 (1990), 84-91.

[14] Schroder, J., Fourth-order two-point boundary value problems; estimates by two side bounds, Nonlinear Anal. 8 (1984), 107-114.

[15] Seda, V., Nieto, J. J., Gera, M., Periodic boundary value problems for nonlinear higher order ordinary differential equations, Appl. Math. Comput. 48 (1992), 71-82.

[16] Usmani, R. A., A uniqueness theorem for a boundary value problem, Proc. Amer. Math. Soc. 77 (1979), 327-335.

[17] Wang, H.-Z., Periodic solutions of four-order differential equations (Chinese), Acta Sci. Natur. Univ. Jilin. 4 (1993), 415-422.

[18] Yang, Y., Fourth-order two-point boundary value problem, Proc. Amer. Math. Soc. 104 (1988), 175-180. 
UPPER AND LOWER SOLUTIONS METHOD FOR FOURTH-ORDER PROBLEMS 61

SHIYOU WENG

General Course Department

Suzhou Vocational University

Suzhou 215104, P. R. China

E-MAIL: WENGSHIYOU2001@YAHOO.COM.CN

DAQING JIANG

School of Mathematics

AND STATISTICS

NoRTHEAST NoRMAL UNIVERSITY

Changlhun 130024, P. R. China

E-MAIL:DAQINGJIANG@VIP.163.COM
HAIYIN GAO

College of Applied Science

ChangChun University

ChangChun, Jilin 130022

P. R. CHINA

E-MAIL: GAOHAIYINHEALTHY@YAHOO.COM

Xuezhang Hou

Mathematics Department

TOWSON UNIVERSITY

BALTIMORE

MARYLAND 21252, USA

E-MAIL: XHOU@TOWSON.EDU 